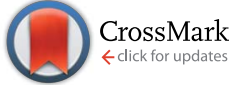

Cite this: J. Mater. Chem. A, 2015, 3, 22915

Received 21st June 2015

Accepted 30th September 2015

DOI: $10.1039 / \mathrm{c} 5 \mathrm{ta} 04551 \mathrm{~g}$

www.rsc.org/MaterialsA

\title{
Diverse isostructural MOFs by postsynthetic metal node metathesis: anionic-to-cationic framework conversion, luminescence and separation of dyes $\uparrow$
}

\begin{abstract}
Soana Seth, Govardhan Savitha and Jarugu Narasimha Moorthy*
An extremely flexible and robust anionic Cd-MOF - accessed with a tetraacid linker, viz., 3,3',5,5'-

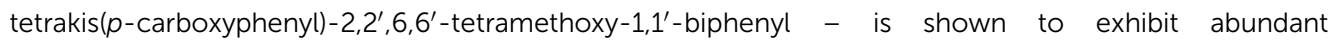
postsynthetic metal exchange (PSME) of metal ion nodes with a number of metal ions that differ vastly in terms of their charges, ionic radii and chemical nature; as many as 16 isostructural new MOFs with transition, lanthanide as well as main group metal ions have been characterized with single crystal X-ray structures determined for 11 of them. The PSME allows unique conversion of the anionic framework structure of the Cd-MOF into a cationic one, when the divalent cadmium metal ions are exchanged by trivalent $\operatorname{Ln}(\mathrm{III})$ ions. The EuaMOF and TbaMOF, accessed by PSME of a transition metal MOF, are shown to be brilliantly luminescent. The switch-over in the charge characteristics of the framework with PSME has been judiciously exploited for the separation of organic dyes based on their charges. The comprehensive investigations illustrate the broad scope of PSME to access new functional materials that cannot be readily accessed.
\end{abstract}

\section{Introduction}

Porous metal-organic frameworks (MOFs) have currently emerged as inextricable hybrid materials with astounding potential for applications in material science, synthetic chemistry and biological sciences. ${ }^{1-14}$ Despite the fact that explosive research over the last two decades has led to well-defined guidelines as to the topologies of MOFs based on connectivity dispositions of the metal ions and the organic linkers, experimental conditions involving solvent, temperature, pressure, additives, etc. render the accessibility of MOFs with predictive topologies a daunting proposition. Key to the success of MOFs for applications is the convenient synthesis with a desired functionality built-in for a specific purpose and the possibility for fine-tuning their properties.,15-18 The synthetic protocols based on de novo approaches entailing judicious choice of organic linkers and metal ions - although successful to some degree - do not lead always to MOFs with predefined topologies/ structures. Post-synthetic modification (PSM) of MOFs has emerged as one of the fine approaches that allows modification

Department of Chemistry, Indian Institute of Technology Kanpur, Kanpur 208016, India. E-mail: moorthy@iitk.ac.in

$\uparrow$ Electronic supplementary information (ESI) available: The X-ray crystal structures and spectral characterization data of $\mathbf{H}_{4} \mathbf{L}$ and intermediates, detailed experimental procedures, plots for the luminescence studies and kinetic studies of dye adsorption and release by the MOFs, crystal data, and TGA and PXRD profiles for all the MOFs. CCDC 1048084-1048088 and 1048244-1048254. For ESI and crystallographic data in CIF or other electronic format see DOI: $10.1039 / \mathrm{c} 5$ ta04551g of the chemical nature and associated functional properties of MOFs in a predictive manner. ${ }^{19-23}$ PSM permits one to access functional MOFs with new properties, while maintaining the same framework topologies of the parent MOFs; such functionally modified MOFs are not necessarily prepared by direct synthetic recipes. ${ }^{24-26}$ The success of PSM, however, is limited by the identification of ideal experimental conditions and the ability of MOFs to sustain crystallinity with the framework structure intact; in some instances, PSM may also lead to unexpected results. ${ }^{27,28}$ The PSM of MOFs may, in general, be carried out in four different ways: (1) by introducing a new functional group via modification of the organic spacer; ${ }^{28-35}(2)$ by anchoring new functionality to the open metal sites in the framework structure; ${ }^{26,36-42}$ (3) by exchanging the guest species in the cavities of the networks; ${ }^{4-48}$ (4) by substituting the building blocks of the framework structures, namely, metal ions and/or the organic ligands, with new building blocks via the metathesis reaction without disturbing the overall integrity of the framework structure. . $^{21,49-52}$

We herein report abundant postsynthetic metal exchange (PSME) of an anionic Cd-MOF with an extremely flexible framework structure-accessed from a twisted $D_{2 \mathrm{~d}}$-symmetric organic spacer $\mathbf{H}_{4} \mathbf{L}$, namely, 3,3',5,5'-tetrakis( $p$-carboxyphenyl)$2,2^{\prime}, 6,6^{\prime}$-tetramethoxy-1,1'-biphenyl, $c f$. Scheme 1 , with transition, lanthanide as well as main group metal ions, leading to a wide array of isostructural MOFs containing different metal ions. ${ }^{53,54}$ As many as 16 isostructural MOFs containing metal ions of varying ionic radii have been characterized, and at least three functional properties are demonstrated. First, the PSME is 


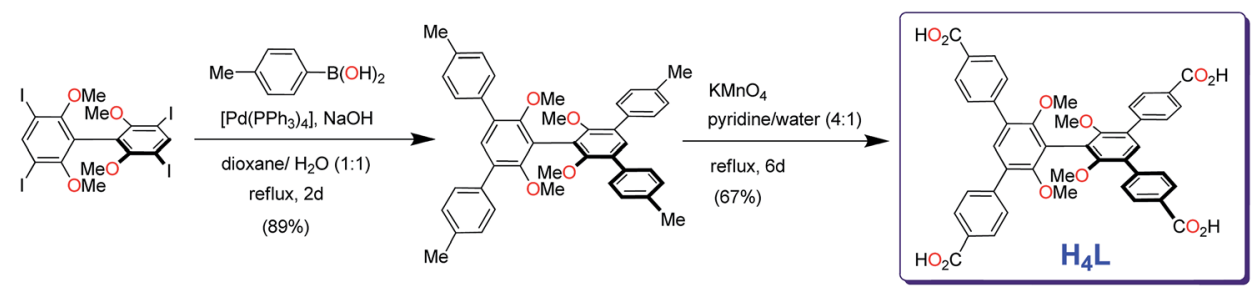

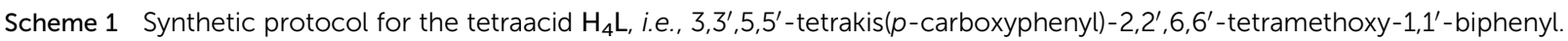

shown to occur in a single crystal-to-single crystal (SCSC) fashion with transition, lanthanide and main group metal ions of very small-to-large ionic radii. Second, the exchange of divalent $\mathrm{Cd}$ (II) ions with trivalent $\mathrm{Ln}$ (III) ions translates essentially into the conversion of the anionic framework into a cationic one, which is extremely rare. ${ }^{49}$ Third, the modified MOFs containing trivalent $\mathrm{Tb}^{3+}$ and $\mathrm{Eu}^{3+}$ are shown to be brilliantly luminescent with green and red emission, respectively, while the parent Cd-MOF exhibits weak linker-based emission. Modification of the luminescence property of a MOF by postsynthetic metal node metathesis is heretofore unknown. The intriguing switchover of the charge characteristics of the framework has been judiciously exploited for remarkable separation of mixtures of organic dyes based on cation/anion exchange. The results compellingly illustrate the power as well as the scope of PSME as an approach to diversifying the unique class of porous hybrid materials, whose structural information is a priori available.

\section{Results and discussion}

The tetracarboxylic acid ligand $\mathbf{H}_{\mathbf{4}} \mathbf{L}$ was prepared by 4 -fold Suzuki coupling of $3,3^{\prime}, 5,5^{\prime}$-tetraiodo-2, $2^{\prime}, 6,6^{\prime}$-tetramethoxybiphenyl with $p$-methylphenylboronic acid, followed by $\mathrm{KMnO}_{4}$ oxidation of the resultant $3,3^{\prime}, 5,5^{\prime}$-tetrakis(4-methylphenyl)-2,2',6,6'-tetramethoxybiphenyl (Scheme 1); the tetraiodo compound was synthesized by following the procedure reported from our laboratory. ${ }^{53 b}$

Heating the solution of $\mathbf{H}_{\mathbf{4}} \mathbf{L}$ in $N, N$-dimethylformamide (DMF) containing four equivalents of $\mathrm{Cd}\left(\mathrm{NO}_{3}\right)_{2}$ in a screw-capped glass vial at $90{ }^{\circ} \mathrm{C}$ for two days led to the formation of colorless crystals of the Cd-MOF. Single crystal structure determination subsequent to intensity data collection and processing revealed that the crystals are centric, and belong to the triclinic crystal system. In the crystal structure, one observes a linear trinuclear $\mathrm{Cd}$ (II) metal cluster with a Cd-Cd-Cd angle of $177.40^{\circ}$, and coordinated by eight carboxylate groups such that it serves as a 8 -connecting node, Fig. 1; the $\mathrm{Cd}-\mathrm{Cd}$ distances between consecutive metal ions are 3.536 and $3.598 \AA$ A. While the central $\mathrm{Cd}(\mathrm{II})$ ion and one of the terminal $\mathrm{Cd}(\mathrm{II})$ ions are found to be octahedral, the other terminal $\mathrm{Cd}(\mathrm{II})$ ion is found to be heptacoordinated with a distorted pentagonalbipyramid geometry. The assembly of an 8-connecting trinuclear cluster and a pair of 3-connecting organic linker $\mathbf{L}$ leads to the 3D framework structure that is overall anionic, Fig. 1. Thus, the repeating unit of the 3D framework of the Cd-MOF is $\left[\mathrm{Cd}_{3} \mathbf{L}_{2}\right]^{2-}$.
The counter cations are inferred to be dimethylammonium species and are not located in the X-ray structure, due supposedly to the disorder arising from their location in the void spaces of the 3D framework. ${ }^{55,56}$ Insofar as the structural aspects of the tetracarboxylate spacer $\mathbf{L}$ are concerned, the central rings of $\mathbf{L}$ are twisted by $65.5^{\circ}$ and $70.6^{\circ}$, while the phenylcarboxylate rings are twisted by angles ranging from $42.8^{\circ}$ to $63.1^{\circ}$ with respect to the dimethoxyaryl rings that constitute the core of $\mathbf{L}$. PLATON analysis suggests that the framework structure is associated with a void volume of $59 \%$, which includes the untraceable dimethylammonium cations. By reducing the organic spacer to a pair of 3-connecting module of the topology of an allene and 8-connecting trinuclear cluster to a simple 8connecting node, one obtains a 3,8-connecting net of sqc-495 topology as that shown in Fig. $1 .^{57}$

In view of the fact that the network is highly porous, we wondered if the cations located in the channels could be replaced by $\mathrm{Cd}^{2+}$ ions themselves by employing a large excess of $\mathrm{Cd}\left(\mathrm{NO}_{3}\right)_{2}$ during the synthesis. Accordingly, solvothermal synthesis of the Cd-MOF was carried out with varying equivalent amounts of $\mathrm{Cd}\left(\mathrm{NO}_{3}\right)_{2}$ with respect to the linker. Thus, a set of five Cd-MOFs were isolated, termed Cd-MOF1, Cd-MOF2, Cd-MOF4, Cd-MOF8 and Cd-MOF12, where the numerical digit(s) refer(s) to the equivalent of $\mathrm{Cd}\left(\mathrm{NO}_{3}\right)_{2}$ employed for the synthesis; in each case, the Cd-MOF was isolated in a respectable yield. In the case of Cd-MOF8 and Cd-MOF12, solventcoordinated $\mathrm{Cd}(\mathrm{II})$ guest ions were found to be present in the cavities of the MOFs - as revealed from single crystal structural analysis, $c f$. ESI $\dagger$ - to counterbalance the negative framework charges; this indeed accounts for different empirical formulae for Cd-MOF8 and 12, which contain $4 \mathrm{Cd}$ (II) ions in contrast to 3 in Cd-MOF1, 2 and 4. All the 5 different MOFs were found to show similar PXRD patterns, see ESI. $\dagger$ The single crystal X-ray structure determination in each of the MOFs revealed intriguingly divergent cell parameters with cell volumes that ranged between 3484 and $3844 \AA^{3}$ for Cd-MOF1, Cd-MOF2 and CdMOF8 and between 7466 and $7589 \AA^{3}$ for Cd-MOF4 and CdMOF12, $c f$. ESI. $\dagger$ The TOPOS analyses showed that the framework structure is similar in all cases, which also accounts for the similarity of all PXRDs. This led us to ponder as to what then is the source of variable cell volumes for Cd-MOF1, 2, 4, 8 and 12. A careful analysis shows that the framework structure is quite flexible and undergoes considerable shrinkage and expansion depending on the matter contained within the framework structure as guest species, namely, counter ions and solvent molecules; the dimethylammonium cations are found to be 
a)

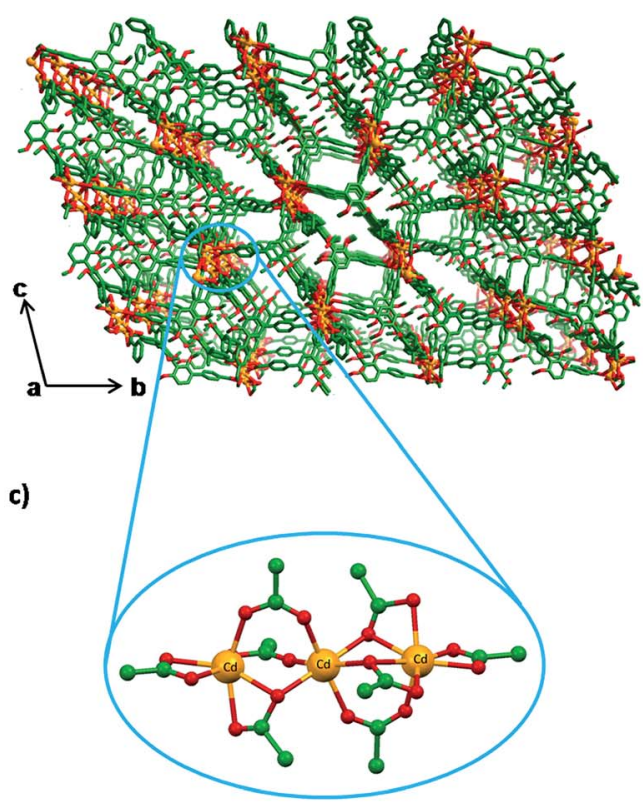

b)

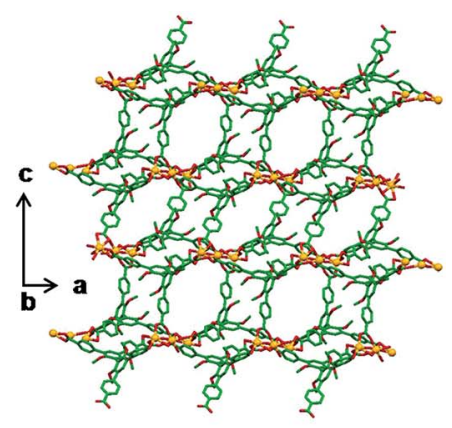

d)

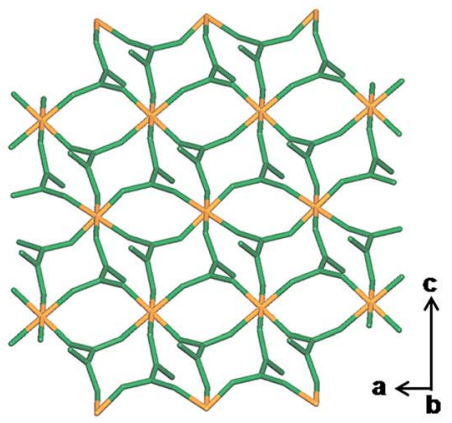

Fig. 1 The crystal packing diagram of the Cd-MOF down a-axis (a) and b-axis (b). Also shown is the coordination around the metal ions in the trimetallic Cd(I) cluster (c). The simplified TOPOS diagram of the crystal packing with the ligand L considered as a pair of 3-connecting spacer and the metallic cluster as a 8-connecting node (d).

replaced by solvent-coordinated Cd(II) ions in Cd-MOF8 and 12 such that the empirical formulae for these cases contain 4 metal ions in contrast to 3 in all others, i.e., Cd-MOF1, 2 and 4, $c f$. ESI. $\dagger$ Thus, we inferred that all of these Cd-MOFs are chemically one and the same. The variations in cell parameters and doubling of the cell volume in some cases are a consequence of guest-induced structural changes of the ligand structure and small deformation of the supposedly linear geometry of the trimetallic cluster, $c f$. ESI, Fig. S1 and S2. $\dagger$ The fact that the tetracarboxylate linker $\mathbf{L}$ lends itself to small structural perturbations is evidenced from considerable torsional flexibility available to carboxyphenyl rings as well as the dimethoxyaryl rings of the core. The dimethoxyaryl rings are found to be twisted by $63-73^{\circ}$, while the angles between the phenylcarboxylate rings and dimethoxyphenyl rings that make up the central core are found to vary between $42.78^{\circ}$ and $82.85^{\circ}, c f$. ESI, Table S2. $\dagger$ It is thus apparent that the flexible framework structure is reproducibly formed with $\mathrm{Cd}\left(\mathrm{NO}_{3}\right)_{2}$, which attests to the fact that the MOF corresponds to the thermodynamic product of the solvothermal synthesis. We were motivated by the remarkable variability and also by the extent of porosity to the framework structure to explore if such a structure allows postsynthetic modification of the cation and metal node; porosity is important for material transport in and out of the crystals.

Remarkably, suspension of crystals of the anionic Cd-MOF4, simply referred to hereafter as Cd-MOF, in $0.01 \mathrm{M}$ solutions of different metal salts in DMF, was found to result in slow exchange of the framework metal ions. With metal ions such as $\mathrm{Cu}(\mathrm{II})$ and $\mathrm{Co}(\mathrm{II})$ ions, the exchange could be made out by visible color changes of the crystals, vide infra. PXRD and SXRD analyses showed that metal ion metathesis occurs leading to new MOFs with the framework integrity intact. The trinuclear cluster of Cd(II) ions was found to be exchanged by a series of transition metal (Tm) ions, viz., $\mathrm{Mn}$ (II), $\mathrm{Co}$ (II), $\mathrm{Cu}$ (II) and Zn(II), to different degrees leading to several Tm@MOFs, vide infra. Indeed, the exchange of $\mathrm{Cd}(\mathrm{II})$ ions was found to proceed in a single crystalto-single crystal fashion for different transition metal ions. Astonishingly, a similar trend was observed for exchange with trivalent lanthanide metal (Ln) ions as well. The exchange was found to occur with a number of lanthanide ions, namely, $\mathrm{La}(\mathrm{III}), \operatorname{Pr}(\mathrm{III}), \mathrm{Nd}$ (III), Eu(III), Tb(III), Gd(III), Sm(III) and $\mathrm{Yb}$ (III), whereby a series of Ln@MOFs is accessed. In the same manner, the framework metal ions were also found to be exchanged by main group metal (Mm) ions, namely, $\mathrm{Mg}$ (II), $\mathrm{Ca}$ (II), $\mathrm{Sr}$ (II) and $\mathrm{Pb}(\mathrm{II})$, leading to unique Mm@MOFs. In Fig. 2 are shown the color changes that one witnesses - as observed under a digital microscope - for cases when the crystals undergo visible color changes.

Our experimentation of postsynthetic metal node metathesis with a number of metal ions led to the characterization of 16 new isostructural MOFs form a single Cd-MOF. The integrity of the framework structure was found to be conserved throughout the metal exchange process as confirmed by powder X-ray diffraction of the resultant MOFs for all, and single crystal structure determinations of several; the X-ray intensity data were collected and structures determined for as many as 11 postsynthetically modified MOFs, $c f$. ESI. $\dagger$ It should be mentioned that our best efforts to independently access all of these MOFs by direct synthesis as that of the Cd-MOF were in 


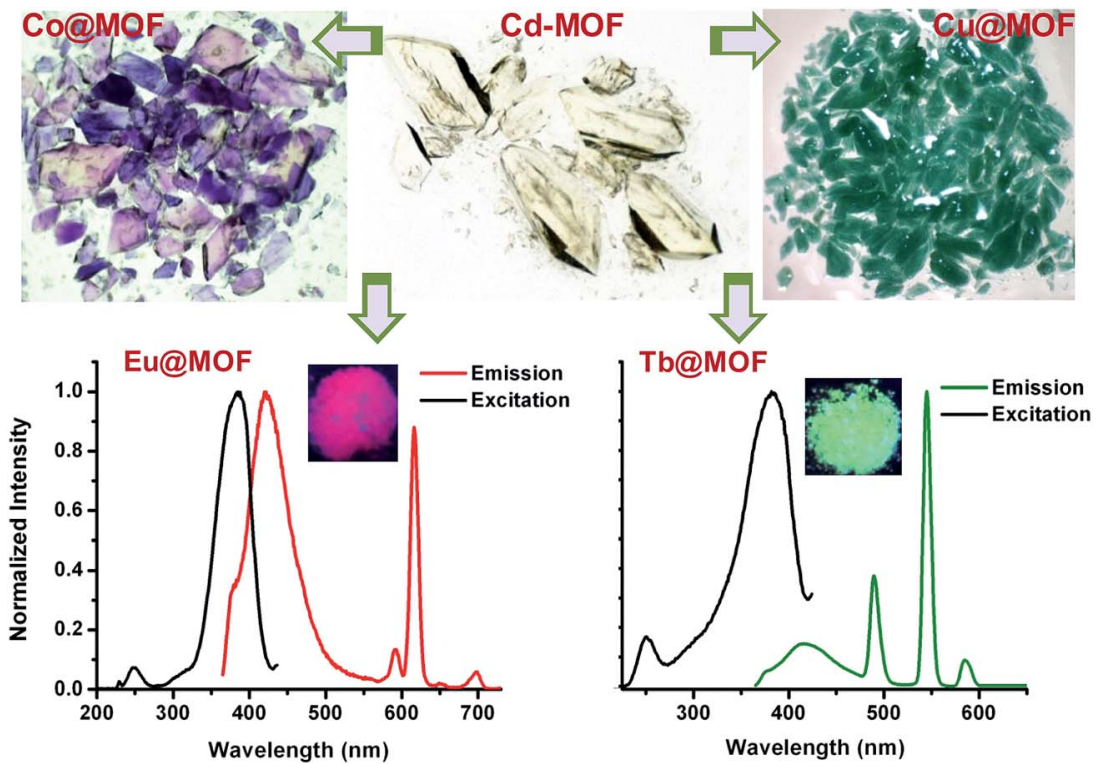

Fig. 2 Visible coloration of the colorless crystals of the Cd-MOF with PSME, and luminescence of Eu@MOF $\left(\lambda_{\mathrm{ex}}=325 \mathrm{~nm}\right)$ and TbaMOF $\left(\lambda_{\mathrm{ex}}=\right.$ $350 \mathrm{~nm}$ )

vain. The extent of metal exchange was evaluated in each case by 3 different analyses, namely, refinement of site-occupancies of the exchanging metal ion and $\mathrm{Cd}(\mathrm{II})$ metal ion, elemental analyses by inductively coupled plasma atomic emission spectroscopy (ICP-AES) and EDX analyses. The results are collated in Table 1 with the cases for which more than $90 \%$ exchange was possible highlighted; it should be noted that the single crystals employed for assessment of exchanged metal ions were not necessarily suspended for the durations similar to those of the samples analyzed by ICP-AES and EDX. Therefore, comparison of the results of EDX/ICP-AES with those of SXRD is not reasonable. Otherwise, it compellingly emerges that the locations of the exchanged metal ions and their contributions can be assessed by the refinement of occupancies that lead to the best R-factor. As is evident from Table 1 , the exchange was found to occur almost quantitatively for $\mathrm{Tb}^{3+}, \mathrm{Yb}^{3+}, \mathrm{Cu}^{2+}, \mathrm{Zn}^{2+}$, $\mathrm{Ca}^{2+}, \mathrm{Sr}^{2+}$ and $\mathrm{Pb}^{2+}$ ions when the crystals were immersed over a period of 25 days. However, $65-75 \%$ exchange was found to occur for $\mathrm{Eu}^{3+}, \mathrm{Nd}^{3+}, \mathrm{Pr}^{3+}, \mathrm{Gd}^{3+}, \mathrm{Co}^{2+}$ and $\mathrm{Mn}^{2+}$ ions after 25 days. The extent of exchange was found to be rather slow amounting to less than $50 \%$ for $\mathrm{La}^{3+}, \mathrm{Sm}^{3+}$ and $\mathrm{Mg}^{2+}$ ions over similar durations. The abundant postsynthetic exchange of framework metal ions is remarkable given the chemical nature and wide range of ionic radii of the metal ions that are employed for exchange. In particular, the exchange with trivalent metal ions is of particular interest, as the framework structure now turns from anionic to cationic for an exchange of $>67 \%$. The counter anions in these cases must be $\mathrm{NO}_{3}{ }^{-}$species, as the lanthanide ions were employed in the form of their nitrates; the latter could not be identified in the crystal structures.

A careful analysis of the X-ray determined structures shows that the trinuclear cluster that is almost linear in the Cd-MOF undergoes significant bending when exchanged by lanthanide metal ions; for transition and main group metal ions, such a deformation is not seen. An overlay of the trimetallic cluster observed in the Eu@MOF and Sm@MOF is shown along with that of the Cd-MOF in Fig. 3. The observed angles between the three metal centers for Ln@MOFs are in the range of 154.7$160.8^{\circ}$. Similarly, the tetracarboxylate linker, as stated at the outset, undergoes torsional motions leading to changes in the

Table 1 Results of analyses of MOFs accessed by PSME of the CdMOF with different metal ions (M), as analyzed by EDX, ICP-AES and SXRD refinement

\begin{tabular}{llll}
\hline & \multicolumn{2}{l}{ M $:$ Cd (atomic ratio) } \\
\cline { 2 - 3 } MOF code & EDX & ICP-AES & SXRD $^{a}$ \\
\hline La@MOF & $62: 38$ & $40: 60$ & $51.6: 48.4$ \\
Pr@MOF & $83: 17$ & $71: 28$ & $52.3: 47.3$ \\
Eu@MOF & $70: 30$ & $68: 32$ & $44: 56$ \\
Nd@MOF & $78: 22$ & $69: 31$ & $55.7: 44.3$ \\
Yb@MOF & $94: 6$ & $93: 7$ & $-{ }^{b}$ \\
Gd@MOF & $86: 14$ & $76: 24$ & $-{ }^{b}$ \\
Tb@MOF & $92: 8$ & $93: 7$ & $100: 0$ \\
Sm@MOF & $74: 26$ & $46: 54$ & $43: 57$ \\
Mn@MOF & $61: 39$ & $65: 35$ & $42: 58$ \\
Co@MOF & $73: 27$ & $90: 10$ & $80.7: 19.3$ \\
Cu@MOF & 100 & $>99$ & $82.8: 17.2$ \\
Zn@MOF & $95.5: 4.5$ & $91: 9$ & $97.3: 2.7$ \\
Mg@MOF & $46: 54$ & $46: 54$ & $-{ }^{b}$ \\
Ca@MOF & $>99$ & $100: 0$ & $43.8: 56.2$ \\
Sr@MOF & $98.5: 1.5$ & $>99$ & $-{ }^{b}$ \\
Pb@MOF & $91: 9$ & $>99$ & $-{ }^{b}$
\end{tabular}

${ }^{a}$ Results obtained by refinement of the atomic positions in the SXRD structure cannot be directly compared with the results of EDX and ICP-AES analyses, as the durations of exchange are different. ${ }^{b}$ The crystals were found to develop cracks to preclude structure determination. Although the cell parameters were determined, structure solution was not possible due to poor intensity data. 


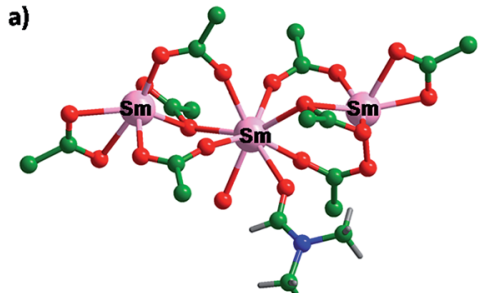

c)

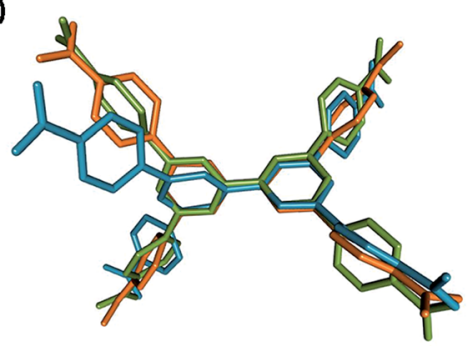

b)

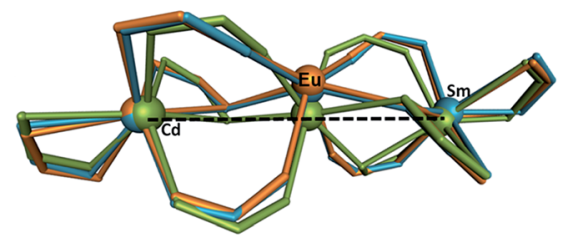

d)

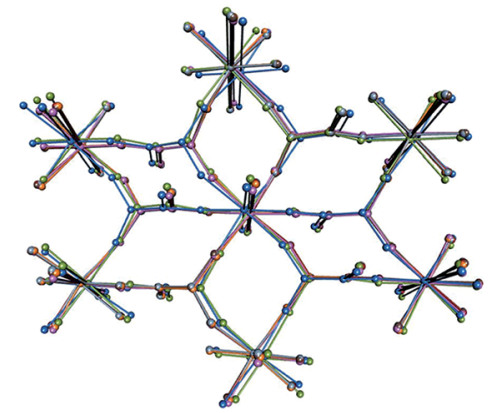

Fig. 3 The coordination environment of the trimetallic cluster in SmaMOF (a). An overlay of the structures of the trinuclear clusters in the CdMOF, SmaMOF and Eu@MOF (b); notice the deviation of the trinuclear centers from linearity in the case of the SmaMOF and Eu@MOF vis-a-vis $\mathrm{Cd}-\mathrm{MOF}$. An overlay of the structures of $\mathrm{L}$ in these three MOFs (c). An overlay of the TOPOS crystal packing for the same three MOFs by reducing the structure to a 3,8-connecting net (d).

overall framework skeleton. In Fig. 3 is also shown an overlay of the structure of $\mathbf{L}$ in the Cd-MOF and two Ln@MOFs. Also notable are small variations in the coordination modes in Ln@MOFs, $c f$. Fig. 3. Solvent molecules are found to remain coordinated to the central metal ions of the trinuclear nodes to satisfy higher coordination numbers of the $\operatorname{Ln}$ (III) ions.

The SC-SC transformation of the Cd-MOF to the MOFs containing other metal ions allows one to modulate the properties of the MOF as a whole, which should be appealing from the point of view of applications notwithstanding incomplete metal exchange. ${ }^{58}$ The anionic framework of the Cd-MOF is converted to the cationic one after exchange of $c a .67 \%$ of the Cd(II) ions by Ln(III) ions. The Ln@MOFs are particularly interesting from the point of view of luminescence exhibited by some of the metal ions in the visible region. The parent Cd-MOF shows very weak ligand-based emission that is blue. However, for the Eu@MOF and Tb@MOF, one observes red and green emissions emanating from the metal ions, while only ligand emission was noted for other Ln@MOFs, $c f$. Fig. 2. It should be mentioned that postsynthetic exchange of SBUs in transition metal-MOFs with lanthanide ions and sequestration of luminescence from the resulting Ln@MOFs are heretofore unprecedented.

Given the ease with which the Cd-MOF permits metal node exchange leading to switchover of the charge characteristics of the framework structure, we envisioned the possibility of exchange of the counter ions with charged organic dyes, whereby separation of organic dyes of different charges should be feasible. ${ }^{59}$ Thus, the parent Cd-MOF with an anionic framework structure and cationic counter ions was explored for exchange with cationic organic dyes, and the Eu@MOF with a cationic framework structure and anionic counter ions was explored for exchange with organic anionic dyes. Methylene blue (MB), thioflavin-T (TT), nile blue (NB) and rhodamine-6G
(Rh6G) were chosen as cationic dyes, and bromophenol blue (BB) and xylene cyanol (XC) were employed as anionic dyes. For competition experiments, neutral nile red (NR) dye was also included. To begin with, crystals of the anionic Cd-MOF were dispersed in $10^{-5}$ to $10^{-6} \mathrm{M}$ solutions of cationic dyes, namely, MB, NB, Rh6G and TT, in DMF. Absorption of the supernatant solution was gradually monitored by UV-vis absorption spectroscopy, $c f$. Fig. 4 and ESI. $\dagger$ The occurrence of cation exchange could be gradually made out from a progressive decrease in the absorbance of the solution in conjunction with coloration of the colorless crystals of the Cd-MOF, Fig. 4. The selectivity of the Cd-MOF to bind cationic dyes in preference to anionic and neutral dyes was investigated by competition experiments. Likewise, the cationic Eu@MOF was also explored for selective binding of an anionic dye in the presence of cationic and neutral dyes, Fig. 4. As expected, the Cd-MOF was found to adsorb the cationic dye MB selectively from a mixture of cationic MB and anionic BB or the neutral NR dyes; indeed, the crystals were found to acquire the color of MB, Fig. 4. Similar selective binding of TT was observed for the pairs of TT and XC, and TT and NR, $c f$. ESI. $\dagger$ Remarkably, the colors of the dyeadsorbed crystals could be reverted to colorless forms by immersing them in a saturated solution of $\mathrm{NaCl}$ in DMF, $c f$. ESI. $\dagger$ In fact, the dye separation process could be carried out multiple times without any noticeable disintegration of the framework structure.

The Eu@MOF - accessed by PSME over a period of $>25$ days was likewise subjected to adsorption of an anionic dye, namely, BB. As shown in Fig. 4, selective adsorption was observed for the anionic dye in the presence of cationic Rh6G dye and neutral NR dye in competition experiments. It should be noted at the same time, no exchange was observed with a Eu@MOF with only ca. $30 \%$ exchange, which attests to the fact that charge reversal as a whole is important. As was observed for Cd-MOF, the colored 

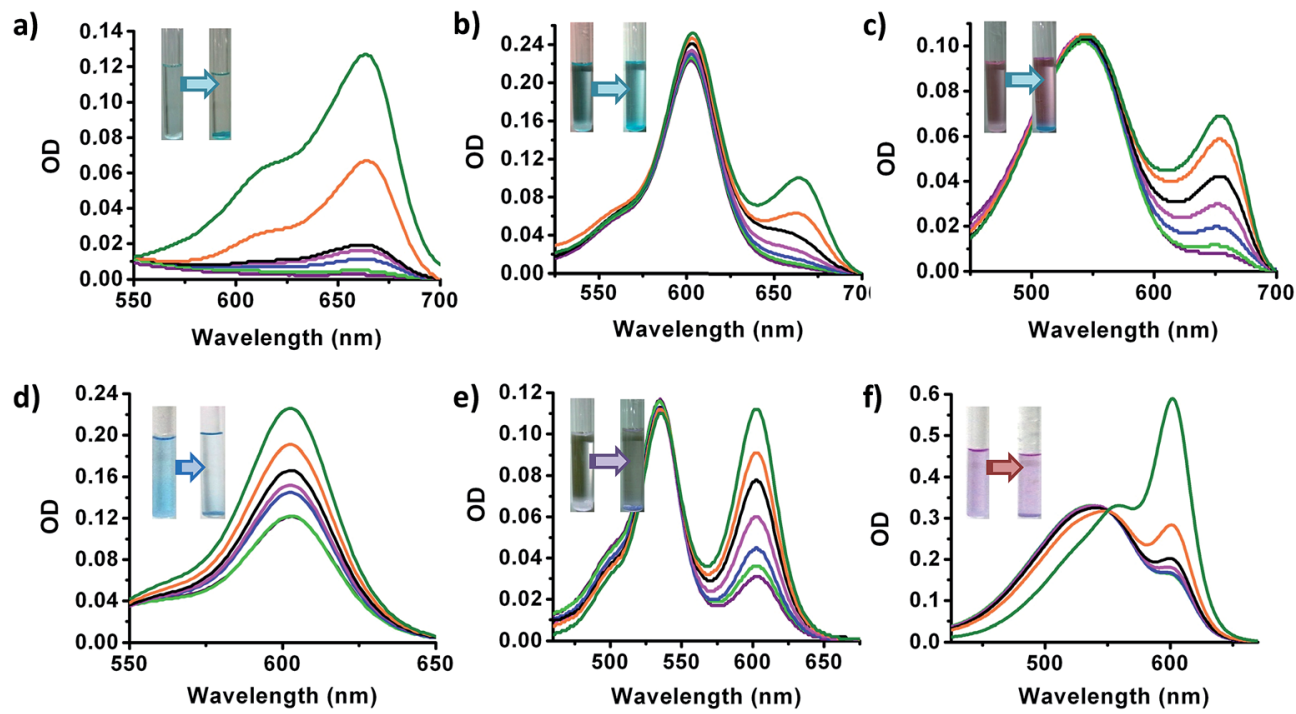

Fig. 4 Adsorption of cationic MB (a) and anionic BB (d) dyes by the anionic Cd-MOF and cationic Eu@MOF, respectively, as monitored by UV-vis absorption spectroscopy. Competitive adsorption of MB in the presence of anionic BB dye (b) and a neutral NR dye by the Cd-MOF (c). Analogous competitive adsorption of BB in the presence of a cationic Rh6G dye (e) and a neutral NR dye (f) by the Eu@MOF. The UV-vis absorption spectra were recorded at times $t=0 \mathrm{~h}$ (olive), $2 \mathrm{~h}$ (orange), $4 \mathrm{~h}$ (black), $8 \mathrm{~h}$ (pink), $14 \mathrm{~h}$ (blue), $24 \mathrm{~h}$ (green) and $48 \mathrm{~h}$ (wine).

dye-adsorbed crystals could be completely reverted to the colorless crystals by suspending them in a saturated solution of $\mathrm{NaCl}$ in DMF. As to the question of how the dye absorption occurs, that is, by migration of the material into the pores or by simple adsorption on the surface, we believe that the latter process is more likely; the sizes of the dyes are larger than the pores for facile transport into the crystals.

It should be mentioned that the rate of PSME of Cd-MOF is rather slow, but its applicability for MOFs of wide ranging metal ions is astounding; the PSME occurs with different lightto-heavy metal ions of varying ionic radii, oxidation states and divergent chemical properties. A thorough literature search reveals that the exchange of the framework metal ions for a given MOF is limited to only a few metal ions, due presumably to the differences in ionic radii, charges and coordination modes. ${ }^{49}$ We are tempted to attribute the abundant PSME of Cd-MOF to the flexible, yet robust integrity of the porous framework structure, which evidently sustains significant structural deformations caused by experimental conditions that entail solvent, guest metal ion concentration, etc. The guest metal ions thus may diffuse in and out of the channels and slowly substitute the $\mathrm{Cd}(\mathrm{II})$ ions that are part of the SBU with the torsional flexibility extant to the ligand $\mathbf{L}$ augmenting the exchange of metal ions under the influence of concentration gradient. The fact that the PSME is largely a consequence of the flexibility of the ligand as well as of the framework structure is corroborated by the reversibility; the metalexchanged MOFs could be reverted back to the Cd-MOF upon suspension of the crystals in solution containing $\mathrm{Cd}\left(\mathrm{NO}_{3}\right)_{2}, c f$. ESI. $\dagger$

Although remarkable flexibility as well as porous nature of the overall framework structure of Cd-MOF lends itself to metal node metathesis and counter ion exchange, the crystals of both Cd-MOF and its metal-exchanged members incidentally were found to be unstable once removed from the solvent, i.e., DMF; in the latter though, the crystals were found to survive for several months. The instability of the crystals to desolvation precluded the otherwise exotic possibility of exploring how reversal in the charge characteristics of the framework structure influences properties such as gas storage, magnetism, etc. of the porous MOFs with the same framework structure.

\section{Conclusions}

$D_{2 \mathrm{~d}}$-symmetric tetratopictetraacid $\mathbf{H}_{\mathbf{4}} \mathbf{L}$ is shown to lead to highly flexible and robust trinuclear Cd-MOF. Significant porosity extant to the MOF structure and flexibility of the organic linker $\mathbf{L}$ are found to permit metal node metathesis with a variety of transition, lanthanide and main group metals ions, providing access to as many as 16 isostructural new MOFs of which 11 metal-exchanged MOFs have been determined by single crystal X-ray structure determinations. The abundant metal exchange with a wide array of metal ions of smaller as well as larger ionic radii than $\mathrm{Cd}(\mathrm{II})$ is unprecedented. The PSME also allows unique conversion of the anionic framework structure of Cd-MOF into a cationic one, when the divalent cadmium metal ions are exchanged by trivalent Ln(III) ions. Eu@MOF and Tb@MOF, accessed by PSME, are shown to be brilliantly luminescent. In addition to metal node metathesis, Cd-MOF and Eu@MOF are demonstrated to be effective for selective and reversible separation of organic dyes based on their charges. The counter ions of anionic Cd-MOF and cationic Eu@MOF are shown to be exchanged selectively by cationic and anionic dyes, respectively, in the presence of neutral dyes or dyes of opposite charges. Remarkable metal node metathesis and cation/anion exchange of a single MOF leading to a wide array of MOFs clearly emphasizes the power of PSME to engineer functional materials with precise structural information. 


\section{Acknowledgements}

JNM is thankful to SERB, India for generous financial support. SS is grateful to CSIR for SPM Senior Research Fellowship. GS is thankful to IITK, Kanpur for institute fellowship. We thank the highly insightful referees for invaluable suggestions in improving the clarity of results.

\section{References}

1 M. Eddaoudi, J. Kim, N. L. Rosi, D. Vodak, J. Wachter, M. O'Keefe and O. M. Yaghi, Science, 2002, 295, 469-472.

2 N. L. Rosi, J. Eckert, M. Eddaoudi, D. T. Vodak, J. Kim, M. O'Keeffe and O. M. Yaghi, Science, 2003, 300, 1127-1129.

3 J. Y. Lee, O. K. Farha, J. Roberts, K. A. Scheidt, S. B. T. Nguyen and J. T. Hupp, Chem. Soc. Rev., 2009, 38, 1450-1459.

4 L. Ma, C. Abney and W. Lin, Chem. Soc. Rev., 2009, 38, 12481256.

5 T. Uemura, N. Yanai and S. Kitagawa, Chem. Soc. Rev., 2009, 38, 1228-1236.

6 R. B. Getman, Y.-S. Bae, C. E. Wilmer and R. Q. Snurr, Chem. Rev., 2012, 112, 703-723.

7 M. P. Suh, H. J. Park, T. K. Prasad and D.-W. Lim, Chem. Rev., 2012, 112, 782-835.

8 K. Sumida, D. L. Rogow, J. A. Mason, T. M. McDonald, E. D. Bloch, Z. R. Herm, T.-H. Bae and J. R. Long, Chem. Rev., 2012, 112, 724-781.

9 J.-R. Li, J. Sculley and H.-C. Zhou, Chem. Rev., 2012, 112, 869932.

10 L. E. Kreno, K. Leong, O. K. Farha, M. Allendorf, R. P. V. Duyne and J. T. Hupp, Chem. Rev., 2012, 112, 1105-1125.

11 D. Liu, K. Lu, C. Poon and W. Lin, Inorg. Chem., 2014, 53, 1916-1924.

12 M. Kurmoo, Chem. Soc. Rev., 2009, 38, 1353-1379.

13 M. Yoon, R. Srirambalaji and K. Kim, Chem. Rev., 2012, 112, 1196-1231.

14 S. Seth, G. Savitha and J. N. Moorthy, Inorg. Chem., 2015, 54, 6829-6835.

15 K. L. Mulfort and J. T. Hupp, J. Am. Chem. Soc., 2007, 129, 9604-9605.

16 Y. K. Hwang, D.-Y. Hong, J.-S. Chang, S. H. Jhung, Y.-K. Seo, J. Kim, A. Vimont, M. Daturi, C. Serre and G. Férey, Angew. Chem., Int. Ed., 2008, 47, 4144-4148.

17 J. An and N. L. Rosi, J. Am. Chem. Soc., 2010, 132, 5578-5579.

18 T. Li, J. E. Sullivan and N. L. Rosi, J. Am. Chem. Soc., 2013, 135, 9984-9987.

19 Z. Wang and S. M. Cohen, J. Am. Chem. Soc., 2007, 129, 12368-12369.

20 Z. Wang and S. M. Cohen, Chem. Soc. Rev., 2009, 38, 13151329.

21 K. K. Tanabe and S. M. Cohen, Chem. Soc. Rev., 2011, 40, 498-519.

22 W. M. Bloch, A. Burgun, C. J. Coghlan, R. Lee, M. L. Coote, C. J. Doonan and C. J. Sumby, Nat. Chem., 2014, 6, 906-912.

23 P. Deria, J. E. Mondloch, O. Karagiaridi, W. Bury, J. T. Hupp and O. K. Farha, Chem. Soc. Rev., 2014, 43, 5896-5912.
24 S. S. Y. Chui, S. M. F. Lo, J. P. H. Charmant, A. G. Orpen and I. D. Williams, Science, 1999, 283, 1148-1150.

25 D. J. Lun, G. I. N. Waterhouse and S. G. Telfer, J. Am. Chem. Soc., 2011, 133, 5806-5809.

26 S. M. Cohen, Chem. Rev., 2012, 112, 970-1000.

27 T. Yamada and H. Kitagawa, J. Am. Chem. Soc., 2009, 131, 6312-6313.

28 S. C. McKellar, A. J. Graham, D. R. Allan, M. I. H. Mohideen, R. E. Morris and S. A. Moggach, Nanoscale, 2014, 6, 41634173.

29 Z. Zhang, L. Zhang, L. Wojtas, P. Nugent, M. Eddaoudi and M. J. Zaworotko, J. Am. Chem. Soc., 2012, 134, 924-927.

30 Z. Zhang, W.-Y. Gao, L. Wojtas, S. Ma, M. Eddaoudi and M. J. Zaworotko, Angew. Chem., Int. Ed., 2012, 51, 9330-9334. 31 T. Gadzikwa, O. K. Farha, K. L. Mulfort, J. T. Hupp and S. T. Nguyen, Chem. Commun., 2009, 3720-3722.

32 K. K. Tanabe, Z. Wang and S. M. Cohen, J. Am. Chem. Soc., 2008, 130, 8508-8517.

33 W. Morris, B. Volosskiy, S. Demir, F. Gándara, P. L. McGrier, H. Furukawa, D. Cascio, J. F. Stoddart and O. M. Yaghi, Inorg. Chem., 2012, 51, 6443-6445.

34 A. M. Shultz, A. A. Sarjeant, O. K. Farha, J. T. Hupp and S. B. T. Nguyen, J. Am. Chem. Soc., 2011, 133, 13252-13255.

35 C. Liu, T. Li and N. L. Rosi, J. Am. Chem. Soc., 2012, 134, 18886-18888.

36 B. L. Chen, M. Eddaoudi, T. M. Reineke, J. W. Kampf, M. O'Keeffe and O. M. Yaghi, J. Am. Chem. Soc., 2000, 122, 11559-11560.

37 S. Q. Ma and H. C. Zhou, J. Am. Chem. Soc., 2006, 128, 1173411735.

38 M. Dincă, A. Dailly, Y. Liu, C. M. Brown, D. A. Neumann and J. R. Long, J. Am. Chem. Soc., 2006, 128, 16876-16883.

39 P. M. Forster, J. Eckert, B. D. Heiken, J. B. Parise, J. W. Yoon, S. H. Jhung, J. S. Chang and A. K. Cheetham, J. Am. Chem. Soc., 2006, 128, 16846-16850.

40 M. Dincă and J. R. Long, Angew. Chem., Int. Ed., 2008, 47, 6766-6779.

41 A. Vimont, J. M. Goupil, J. C. Lavalley, M. Daturi, S. Surble, C. Serre, F. Millange, G. Férey and N. Audebrand, J. Am. Chem. Soc., 2006, 128, 3218-3227.

42 Y. K. Hwang, D. Y. Hong, J. S. Chang, S. H. Jhung, Y. K. Seo, J. Kim, A. Vimont, M. Daturi, C. Serre and G. Férey, Angew. Chem., Int. Ed., 2008, 47, 4144-4148.

43 M. Dincă and J. R. Long, J. Am. Chem. Soc., 2007, 129, 1117211176.

44 F. Nouar, J. Eckert, J. F. Eubank, P. Forster and M. Eddaoudi, J. Am. Chem. Soc., 2009, 131, 2864-2870.

45 H. Fei, D. L. Rogow and S. R. J. Oliver, J. Am. Chem. Soc., 2010, 132, 7202-7209.

46 Z. Zhang, W.-Y. Gao, L. Wojtas, S. Ma, M. Eddaoudi and M. J. Zaworotko, Angew. Chem., Int. Ed., 2012, 51, 9330-9334.

47 J. An, C. M. Shade, D. A. Chengelis-Czegan, S. Petoud and N. L. Rosi, J. Am. Chem. Soc., 2011, 133, 1220-1223.

48 B. Li, Y. Zhang, D. Ma, T. Ma, Z. Shi and S. Ma, J. Am. Chem. Soc., 2014, 136, 1202-1205.

49 S. Das, H. Kim and K. Kim, J. Am. Chem. Soc., 2009, 131, 3814-3815. 
50 T. Li, M. T. Kozlowski, E. A. Doud, M. N. Blakely and N. L. Rosi, J. Am. Chem. Soc., 2013, 135, 11688-11691.

51 M. Lalonde, W. Bury, O. Karagiaridi, Z. Brown, J. T. Hupp and O. K. Farha, J. Mater. Chem. A, 2013, 1, 5453-5468.

52 C. K. Brozek and M. Dincă, Chem. Soc. Rev., 2014, 43, 54565467.

53 For our earlier work on MOFs based on analogous twisted tetrapyridyl ligands, see: (a) R. Natarajan, G. Savitha, P. Dominiak, K. Wozniak and J. N. Moorthy, Angew. Chem., Int. Ed., 2005, 44, 2415-2419; (b) S. Seth, P. Venugopalan and J. N. Moorthy, Chem.-Eur. J., 2015, 21, 2241-2249.

54 For our recent studies postsynthetic site-selective metal exchange in a single crystal-to-single crystal fashion, see: A. Bajpai, P. Chandrasekhar, G. Savitha, R. Banerjee and J. N. Moorthy, Chem.-Eur. J., 2015, 21, 2759-2765.
55 J. An and N. L. Rosi, J. Am. Chem. Soc., 2009, 131, 83768377.

56 J. Yu, Y. Cui, C. Wu, Y. Yang, Z. Wang, M. O'Keeffe, B. Chen and G. Qian, Angew. Chem., Int. Ed., 2012, 51, 1054210545.

57 R.-L. Chen, X.-Y. Chen, S.-R. Zheng, J. Fan and W.-G. Zhang, Cryst. Growth Des., 2013, 13, 4428-4434.

58 X.-J. Wang, P.-Z. Li, L. Liu, Q. Zhang, P. Borah, J. D. Wong, X. X. Chan, G. Rakesh, Y. Lia and Y. Zhao, Chem. Commun., 2012, 48, 10286-10288.

59 For recent studies of selective dye adsorption, see: (a) C.-Y. Sun, X.-L. Wang, C. Qin, J.-L. Jin, Z.-M. Su, P. Huang and K.-Z. Shao, Chem.-Eur. J., 2013, 19, 3639-3645; (b) Y.-C. He, J. Yang, W.-Q. Kan, H.-M. Zhang, Y.-Y. Liu and J.-F. Ma, J. Mater. Chem. A, 2015, 3, 1675-1681. 